\title{
LORSQUE (SE) VOIR NE VOIT PLUS
}

\section{INTRODUCTION}

Il n'est pas surprenant que parmi les verbes de perception, qui forment un champ sémantique riche et ramifié, le verbe voir ait une place à part. Cela est dû bien évidemment au rôle cognitif primordial joué par la vision et cette place privilégiée se reflète dans la langue, essentiellement au niveau lexical, mais également dans le système grammatical. Le verbe (se) voir, verbe de perception visuelle à sens résultatif, est le seul, avec $(s$ ') entendre, qui franchit la frontière séparant le lexique de la grammaire. Certains emplois de ce verbe présentent des exemples du processus de grammaticalisation transformant une unité lexicale en une unité grammaticale.

L'article se limitera à l'étude des constructions où (se) voir est suivi d'un complément infinitif. Pour les besoins de l'analyse, on s'intéressera également aux verbes $(s$ ')entendre et (se) sentir, pour montrer que ce phénomène n'est pas une caractéristique propre à tous les verbes de perception à sens résultatif. Dans un premier temps, nous nous intéresserons à la sémantique et aux différents emplois du verbe, notamment en termes de désémantisation et de grammaticalisation. Ensuite nous proposerons une analyse des constructions (se) voir + INF qui s'appuiera sur la notion de subjectivation (ou subjectivisation), telle qu'elle a été élaborée par R. L. Langacker (1991, 1999).

\section{DE LA DÉSÉMANTISATION À LA GRAMMATICALISATION}

La grammaticalisation est un processus continu et graduel au cours duquel le sens lexical évolue vers un sens grammatical, plus abstrait et plus schématique ${ }^{1}$. Ce processus franchit plusieurs étapes de désémantisation permettant au futur élément grammatical d'être capable de s'adapter à un grand nombre de contextes très divers ne se pliant qu'à des contraintes de

1 Voici la définition qu'en proposent P. Hopper et E. Traugott et qui est bien adaptée au traitement du verbe (se) voir puisqu'elle ne conçoit pas la grammaticalisation comme un phénomène discret ne connaissant que deux valeurs opposées, mais comme un phénomène continu où différentes valeurs s'échelonnent entre deux extrêmes. La grammaticalisation est « that subset of linguistic changes through which a lexical item in certain uses becomes a grammatical item, or through which a grammatical item becomes more grammatical. » (Hopper/Traugott 1993 : 2) 
nature morpho-syntaxique. Pour le verbe voir, la désémantisation consiste en un affaiblissement progressif du trait sémantique /perception/ qui permet de transformer un verbe à sens lexical en un auxiliaire.

Prenons à titre d'exemple l'analyse du verbe voir proposée par J. Picoche (1986, 2529) dans une perspective guillaumienne. L'analyse se fonde sur les notions de cinétisme et de subduction. Le cinétisme de voir, à l'origine de sa polysémie, se répartit, selon Picoche, en cinq types d'emplois correspondant aux cinq étapes de subduction, menant de la signification « plénière » aux significations « subduites ».

- emplois de nature nettement sensorielle :

(1) De ma fenêtre je vois le port.

- emplois de nature entre sensorielle et non sensorielle :

(2) Il s'est vu décerner les palmes académiques.

- emplois de nature non sensorielle :

(3) Des situations de ce genre, on en voit dans tous les bureaux d'aide social.

- emplois de nature non sensorielle à activité d'esprit croissante :

(4) Sa façon de voir n'est pas la mienne.

- emplois « subduits », de nature non sensorielle à activité d'esprit nulle

\section{(5) Saint-Malo a vu naître Chateaubriand.}

La désémantisation est nettement moins avancée dans le cas de ( $\left.s^{\prime}\right)$ entendre et de (se) sentir. Tous ces verbes, et notamment leurs formes pronominales, ont cependant développé des emplois produisant différents « effets » de diathèse. Dans les exemples (2) et (5) cités ci-dessus, ce sont le bénéficiaire et le lieu où s'est produit l'événement qui assument la fonction de sujet, même si ces deux actants occupent «normalement » la place de complément d'objet indirect et de complément circonstanciel de lieu :

(2') On lui a décerné les palmes académiques.

(5') Chateaubriand est né à Saint-Malo. 
Les réaménagements des actants relevant de la diathèse et répondant ainsi aux besoins des locuteurs s'observent également avec les deux autres verbes, bien que ce réaménagement semble moins radical :

(6) J'ai entendu dire qu'il est très malade.

(6') J'ai entendu qu'on disait qu'il était très malade.

(7) La mère de Nicolas s'est entendu reprocher d'avoir mis le sel pour les œufs durs.

(7') On a reproché à la mère de Nicolas d'avoir mis le sel pour les œeufs durs.

(8) J'ai senti le sol s'effondrer en miettes.

(8') J'ai senti que le sol s'effondrait en miettes.

(9) Elle s'est senti mordre par le chien².

(9') Elle a senti que le chien la mordait.

La plupart des grammaires s'accordent pour voir dans l'emploi désémantisé de la forme pronominale se voir un auxiliaire de passivation ${ }^{3}$. Le verbe s'entendre fait moins l'unanimité : il n'est, à notre connaissance, cité que dans Grammaire méthodique (Riegel, Pellat, Rioul 1994, 442-443), avec se faire, se laisser et se voir. Se sentir est absent des grammaires.

Selon bon nombre de linguistes (François 2000), l'emploi de se voir, de même que de se faire, comme auxiliaires de diathèse (voir plus loin) est favorisé par le fait que le français, à la différence de l'anglais par exemple (Einstein was offered the presidency of Israel), ne permet pas de passif du bénéficiaire/détrimentaire : ces auxiliaires semblent pallier cette « insuffisance » (Einstein s'est vu/fait offrir la présidence d'Israël.).

\section{SUBJECTIVATION}

La subjectivation (ou subjectification, ang. subjectification) dans le sens langackerien du terme (Langacker 1991, 1999) présente un type de changement sémantique qui entre dans le processus de désémantisation et peut mener jusqu'à la grammaticalisation plus ou moins

2 Cet exemple figure dans la rubrique Difficultés du Dictionnaire Larousse en ligne (http://www.larousse.com/ fr/dictionnaires/francais/sentir/72147/difficulte ; consulté le 10 avril 2015) pour illustrer les difficultés de l'accord du participe passé. Le corpus sur lequel je me base (voir plus loin) ne comporte aucune occurrence de la construction où les sujets de se sentir et de l'infinitif sont différents.

3 La tradition grammaticale préfère qualifier ces auxiliaires, normalement suivi d'un infinitif, de même que des auxiliaires d'aspect ou de temps, de semi-auxiliaires, les distinguant ainsi des auxiliaires avoir et être, suivis de participes et pleinement grammaticalisés. 
complète d'une unité lexicale 4 . Cette notion ne doit pas être rattachée aux notions d'expressivité ou d'affectivité. Elle doit être de plus distinguée de la définition qu'en propose E. C. Traugott (2010) dans le cadre de son approche sémantico-pragmatique privilégiant essentiellement l'axe diachronique.

La subjectivation langackerienne est étroitement liée au phénomène dit de grounding, donc à l'ancrage situationnel d'une expression linguistique (mot, syntagme, énoncé...). Toute expression linguistique, quelque décontextualisée qu'elle soit, est arrimée à un « fond» (ang. ground) que constituent les participants de la communication, en premier lieu les interlocuteurs, et la situation ou le contexte impliquant le lieu et le temps de l'interaction. Dans les syntagmes nominaux, ce sont les déterminants qui assurent cet ancrage ( $l a$ maison), pour les verbes conjugués à la forme personnelle, ce sont le temps et le mode ( $\mathrm{Il}$ est sorti / Qu'il sorte). Chaque expression linguistique présente une asymétrie entre, d'un côté, un conceptualisateur dont le rôle prototypique est d'être pleinement subjectif et de ne pas participer à l'événement désigné par l'objet et, de l'autre, un objet de conceptualisation qui doit être profilé et pleinement objectif. Or, il est rare que cette asymétrie soit pleinement réalisée :

\section{(10) Pierre sort de la chambre.}

Bien que le conceptualisateur reste « hors-scène », sa subjectivité transparaît au travers le présent de l'indicatif de sort et l'article défini du groupe nominal la chambre : ces éléments arriment l'énoncé à la situation et aux participants de l'énonciation.

Il se peut que cette asymétrie soit plus radicalement diminuée, voire brisée. Dans un premier cas de figure, elle s'estompe au profit d'une sorte d'objectivation. Lorsque nous utilisons un élément déictique, par exemple, la perspective change : cette fois, c'est le conceptualisateur (je) ou un des éléments directement attaché à lui (ici) qui sont mis au-devant de la scène. Ces éléments deviennent des objets de conceptualisation :

(11) Je suis devant la porte.

\section{(12) C'est la tradition ici.}

Dans un deuxième cas de figure, on passe d'une conception relativement objective d'une entité à une conception plus subjective. En d'autres mots, c'est la subjectivité du conceptualisateur qui se met progressivement à la place de l'objectivité de l'entité perçue. Dans ce cas-là, on parle de subjectivation. Prenons l'exemple du verbe aller en tant qu' auxiliaire.

4 Bien que les phénomènes de grammaticalisation soient essentiellement étudiés dans des perspectives diachroniques, la notion de subjectivation permet également l'adoption d'une perspective synchronique ne tenant pas compte de l'ordre historique de l'apparition des attestations. 
(13) Jean va à Paris.

\section{(14) Jean va écrire sa communication.}

Dans (13), aller garde son sens concret. Le rôle du conceptualisateur y est minimal et se réduit au balayage séquentiel de l'événement conçu de façon objective. Le conceptualisateur n'a aucune difficulté à identifier le trajecteur (ang. trajector) (Jean), le procès (aller) et la direction qui constitue le repère (Paris).

L'exemple (14) est plus complexe. Le verbe aller passe du domaine spatial au domaine temporel, plus abstrait. L'énoncé ne décrit aucun mouvement concret, mais implique un mouvement abstrait : le conceptualisateur remplace le mouvement spatial par une sorte de projection dans le futur. C'est donc le conceptualisateur qui « reconstitue » mentalement et de façon subjective ce mouvement, son trajecteur et son repère, qui n'est plus constitué par une entité concrète, mais par le procès désigné par la construction infinitive. La subjectivation de aller peut aboutir à un haut degré de grammaticalisation, ce qui semble être confirmé par les contraintes syntaxiques pesant sur le choix des temps verbaux : seuls le présent et l'imparfait sont admis. Le futur simple ou le passé composé, par exemple, restituent le sens spatial « objectif »:

(14') Jean ira présenter sa communication.

(14') Jean est allé présenter sa communication.

\section{SUBJECTIVATION ET ATTÉNUATION DU SENS}

Pour déterminer le degré de subjectivation, nous nous fonderons sur deux types de critères qui rendront compte de l'atténuation sémantique 5 .

Le premier type de critères évaluera le contrôle exercé par le sujet du verbe de perception : dans quelle mesure ce sujet reste-t-il le siège d'action, d'expérience ou de responsabilité du procès. L'atténuation progressive du sens s'accompagne notamment d'une dissipation de ce contrôle.

Le deuxième type de critères concerne le changement de domaine dans lequel s'inscrit le procès. On peut observer deux cas de figure : soit le verbe change de domaine de perception, soit un domaine de perception est remplacé par un domaine plus abstrait.

5 L'analyse proposée s'appuie essentiellement sur deux corpus de textes journalistiques : Le Monde 2002 accessible par le concordancier Web Le Migou proposé par l'Observatoire de linguistique Sens-Texte (http://olst.ling. umontreal.ca/migou/) et les Archives du Monde diplomatique 1954-2011 sur DVD-ROM (Le Monde diplomatique SA, IDM, 2012). 


\section{$5 \quad V O I R+I N F I N I T I F$}

L'analyse des exemples relevés dans le corpus semble confirmer que voir parvient à un haut degré de subjectivation. Les deux autres verbes de perception que nous avons mentionnés en introduction (entendre, sentir) n'y parviennent pas. Dans (15) le sujet reste le siège de l'action de perception et exerce un contrôle parfait du procès.

(15) En même temps, nous avons vu une fumée noire sortir de la base de la montagne, se développant sans cesse. [Le Migou-Le Monde 2002]

Dans l'exemple (16), le verbe voir ne relève plus du domaine de perception visuelle, mais plutôt d'une perception nettement plus abstraite :

(16) [L]e tourisme aurait vu ses chiffres dopés par la réservation d'un million et demi de séjours supplémentaires, dont la moitié par des touristes étrangers. [Le Migou-Le Monde 2002]

Dans (17), le domaine dont relève voir n'est plus celui d'une perception quelconque.

(17) Jadis occupée par les Ottomans, cette ville de 18000 âmes qui vit naître aussi Enver Hoxa.[Le Monde diplomatique, avril 1997]

Si une lecture métonymique semble encore possible pour le sujet dans (16) (tourisme = responsables de ce secteur d'activité), ce qui permet à ce sujet de rester le siège d'expérience, voire de responsabilité, exerçant encore un certain contrôle, cette lecture semble peu convaincante pour le sujet dans (17) (ville = les habitants de la ville ?).

Comparons voir aux verbes entendre et sentir pour montrer que ce phénomène ne touche pas tous les verbes de perception.

Le verbe entendre ne quitte jamais le domaine de perception auditive et le sujet exerce pleinement le contrôle de l'activité exprimée par le verbe, comme dans l'exemple (18).

(18) Paquita n'a jamais entendu son père parler autant. [Le Monde diplomatique, mars 1993]

Le cas de sentir est plus complexe, ce qui est dû à sa grande polysémie : ce verbe peut couvrir tous les types de perception. Les deux exemples suivants illustrent bien ces passages du plus concret vers plus abstrait :

(19) Elle sentait une chaleur rouge lui parcourir le corps. [Le Monde diplomatique, septembre1998] 
(20) Mario sentit sa voix s'enrouer et ses yeux se mouiller. [Le Monde diplomatique, février 1987]

(21) Le gouvernement Prodi sent sa fin approcher. [Le Monde diplomatique, avril 2008]

Les emplois métaphoriques et plus ou moins figés sont fréquents :

(22) Tout autre que cet étonnant jésuite, qui devait faire la paix entre la Pologne et la Russie, eût senti son sang se glacer en pénétrant dans le palais d'Ivan le Terrible. [Le Monde diplomatique, mars 1970]

(23) Les Etats-Unis n'ont pas senti le vent tourner - ou l'ont ignoré - laissant un accident déclencher un mouvement qui va au-delà de la question du statut des forces américaines. [Le Migou-Le Monde 2002]

Le degré de subjectivation est cependant faible, puisque le sujet continue d'exercer son contrôle et reste le siège d'une action dirigée vers l'objet de la perception qui se constitue autour du processus exprimé par l'infinitif.

\section{AUXILIARE DE DIATHÈSE}

Les auxiliaires de diathèse, terme qu'on préfère à " auxiliaires de passivation », participent au réaménagement des actants en fonction des besoins communicatifs des locuteurs (cf. Muller, 2005). Du point de vue cognitif, on observe que, dans les constructions « auxiliaire de diathèse + infinitif », le trajecteur du verbe conjugué est toujours identique au trajecteur du procès exprimé par l'infinitif. L'analyse qui établit des parallèles entre ce schéma cognitif unique et les constructions syntaxiques permet de dégager plusieurs cas de figures.

Dans un premier cas de figure les sujets des verbes sont identiques $\left(\mathrm{S}_{\text {pers }}=\mathrm{S}_{\text {inf }}\right)$. Les exemples de ce type sont très rares pour le verbe se voir, mais également pour s'entendre

(24) Sur RTL, pourtant, elle vante le bilan du gouvernement à l' égard des femmes et explique qu'elle "ne se serait pas vue épouser un homme austère ». [Le Migou-Le Monde 2002]

(25) À Antony qui contestait la véracité des faits et qualifiait son interlocuteur de «cochon 》, " vendu », " voyou » et le priait de sortir " s' il avait des couilles 》, Marine Le Pen s'est entendu lancer cette remarque : "Je sais que cela t'aurait fait plaisir que je dise cela, en l' occurrence, je n' ai pas envie de te draguer. » [Le Migou-Le Monde 2002] 
Les occurrences de la construction « se sentir + infinitif » sont tout aussi rares. On observe que tous les exemples trouvés dans les deux corpus relèvent de ce premier cas de figure (voir pourtant l'exemple (9) ci-dessus et la note 2). Le potentiel de diathèse de se sentir est donc beaucoup trop faible pour qu'on puisse parler d'un auxiliaire de diathèse.

(26) D’ici trois mois, elle sera guérie. Elle se sentira renaître! [Le Monde diplomatique, novembre 1982]

Le deuxième cas de figure regroupe des constructions où le sujet du verbe conjugué est coréférentiel au COD de l'infinitif $\left(\mathrm{S}_{\text {pers }}=\mathrm{COD}_{\text {inf }}\right)$. Les occurrences de ce type pour le verbe se voir sont rares.

(27) Goldman Sachs s'est vu accuser par la SEC d'avoir trompé ses clients en leur vendant en 2007 des collateralized debt obligations (CDO), produits dérivés complexes adossés à des crédits immobiliers à risque (subprime), sans les informer qu'elle pariait dans le même temps sur leur chute. [Le Monde diplomatique, août 2010]

Il en est de même pour s'entendre. Pour se sentir nous n'avons pas trouvé d 'occurrence dans nos corpus.

(28) Quand il s'est entendu traiter de „nazi“, le secrétaire d'Etat au Logement, Alex Goldfarb, s'est emporté. [Le Monde diplomatique, août 1995]

Le dernier cas de figure étudié, présentant des constructions où le sujet du verbe conjugué est coréférentiel au COI de l'infinitif $\left(\mathrm{S}_{\text {pers }}=\mathrm{COI}_{\text {inf }}\right)$, regroupe l'écrasante majorité des exemples relevés. La fréquence relative de ce type de constructions corrobore la thèse, déjà évoquée ci-dessus, selon laquelle les constructions se voir + infinitif, de même que se faire + infinitif, sont des « constructions de remplacement » qui pallient l'absence, en français, d'un passif du destinataire.

Les infinitifs les plus fréquents se combinant avec se voir sont attribuer, autoriser, confier, confirmer, décerner, interdire, proposer, refuser.

(29) La société devra verser des indemnités allant de 17000 à 60000 euros par ouvrier, tandis que le repreneur frauduleux se verra infliger un an de prison. [Le Monde diplomatique, février 2010]

Se voir semble accepter également des infinitifs impliquant d'autres types de perception, notamment la perception auditive (demander, dire, proposer, répondre, reprocher). 
(30) Demandant aux soldats israéliens postés au bord de la route de le protéger, il se serait vu répondre par l'un d'entre eux : "Si tu restes ici, je te tire dessus ». [Le Migou-Le Monde 2002]

Pour le verbe s'entendre, on a relevé les combinaisons avec conseiller, demander, dire, expliquer, répéter, reprocher, riposter. Tous ces verbes impliquent une perception auditive, ce qui signale un degré de subjectivation moins élevé.

(31) Président sud-coréen et lauréat du prix Nobel de la paix, M. Kim Dae-jung s'est entendu dire lors d'une rencontre avec le président Bush, en mars 2001, que le leader nordcoréen n'était pas digne de confiance. [Le Monde diplomatique, février 2003]

\section{CONCLUSIONS}

Dans le prolongement des analyses proposées dans les sections 5 et 6 , on peut faire remarquer que se voir, de même que voir, manifeste un degré très élevé de subjectivation. Se voir accepte le changement de domaine de perception et même « son atténuation » au profit d'un domaine plus abstrait. On observe également que le sujet peut perdre une grande partie du contrôle du procès. Dans ce cas-là, il cesse d'être " pleinement » un siège d'action, d'expérience ou de responsabilité. Souvent, seule une lecture métonymique permet de lui réattribuer une partie du contrôle perdu, comme c'est le cas dans l'exemple ci-dessous :

(32) Les compagnies minières (souvent multinationales) qui versent, sur la base du volontariat, cette contribution supplémentaire de deux ans se sont vu garantir le prolongement d'une des redevances les plus faibles du monde jusqu'en 2025 ! [Le monde diplomatique, mai 2011]

Nous avons déjà observé que les degrés de subjectivation de sentir et d'entendre restent très faibles. La même observation s'impose aussi pour les variantes pronominales des deux verbes. Ni se sentir ni s'entendre n'admettent de changement de domaine de perception. Les sujets des deux verbes contrôlent l'action en tant que sièges d'action ou d'expérience. De plus, seul s'entendre se prête à de plus importants effets de diathèse.

L'analyse proposée montre que seul se voir pourrait être qualifié d'auxiliaire de diathèse. Son degré de subjectivation reste cependant inférieur au degré de subjectivation de l'auxiliaire être. Citons deux remarques qui sont souvent évoquées dans la littérature sur le sujet et qui prouvent que la subjectivation de se voir est loin d'être achevée et qu'il y reste toujours des traces de son sémantisme de base.

- selon la plupart des linguistiques, se voir s'accommoderait mal de certains contextes qui s'opposent radicalement à son sémantisme de base : 
(33) Cet étudiant s'est vu attribuer, en son absence, le premier prix de la Faculté par le Doyen. (Bat-Zeew Shyldkrot 1981 : 398)

- se voir n'est en règle générale pas substituable à se faire lorsque celui-ci dénote un procès intentionnel, de plus l'emploi de se voir est réservé aux registres plus formels, plus soignés (voir François $2000: 170-173$ ).

(34) Et il a dit qu'il s'en lavait les mains. Que... - Encore un s'est fait ['vu] raccrocher au nez, le docteur Martin (François $2000: 171$ )

(35) Mais, dans toute cette histoire, on s'est fait [ $\left.{ }^{*} v u\right]$ baiser de A à Z. (François 2000 : 171)

En conséquence, il serait plus prudent et plus judicieux de réintroduire le terme de « semi-auxiliaire » qui mettrait en évidence le degré de subjectivation distingant se voir de être, seul « vrai » auxiliaire de diathèse.

\section{BIBLIOGRAPHIE}

BAT-ZEEV SHYLDKROT, Hava (1981) À propos de la forme passive 'se voir $+\mathrm{V}_{\text {inf }}$ '. Folia Linguistica XV/3-4, 387-306.

DEFRANCQ, Bart (2000) Approche contrastive des (semi-) auxiliaires du passif de l'objet prépositionnel ». Études romanes 45, 185-204.

FRANÇOIS, Jacques (2000) Désémantisation verbale et grammaticalisation : (se) voir employé comme outil de redistribution des actants. Syntaxe et Sémantique 2, 159-175.

HOPPER, Paul J./Elizabeth CLOSS TRAUGOTT (1993) Grammaticalization. Cambridge : Cambridge University Press.

LANGACKER, Ronald W. (1991) Concept, Image and Symbol: The Cognitive Basis of Grammar. Berlin, New York : Mouton de Gruyter.

LANGACKER, Ronald W. (1999) Grammar and Conceptualization. Berlin, New York : Mouton de Gruyter.

MULLER, Claude (2005) Diathèse et voix en français. F. Hrubaru (éd.), Interaction entre sémantique et pragmatique. Bucarest : Editura ASE, 73-95.

PICOCHE, Jacqueline (1986) Structures sémantiques du lexique français. Paris : Nathan.

RIEGEL, Martin/Jean Christophe PELLAT/René RIOUL (1994) Grammaire méthodique du français. Paris : PUF.

TRAUGOTT, Elizabeth Closs (2010) Revisiting subjectification and intersubjectification. Kristin Davidse/Lieven Vandelanotte/Hubert Cuyckens (éds.), Subjectification, Intersubjectification and Grammaticalization. Berlin, New York : Mouton de Gruyter, 29-70. 


\title{
POVZETEK
}

\section{Ko (se) voir ne vidi več}

Članek rabo glagola (se) voir analizira kot primer semantične kategorije subjektivizacije, kot jo je razvil R. W. Langacker. Subjektivizacija označuje semantično spremembo, ki vsebuje pomensko slabljenje, ki lahko vodi do gramatikalizacije. Bolj objektiven način konstruiranja entitete nadomesti bolj subjektiven način. Povedano drugače, subjektivizacija je proces, v katerem je objektivnost konstruirane entitete postopno nadomeščena s subjektivnostjo konceptualizatorja. Glagol (se) voir v primerjavi z ostalimi glagoli zaznave ( $\left(s^{\prime}\right)$ entendre, (se) sentir) doseže visoko stopnjo subjektivizacije. $(\mathrm{Se})$ voir lahko zamenja konkretno pomensko področje zaznave in celo pomensko področje zaznave nadomesti s kakšnim bolj abstraktnim področjem. Istočasno slabi osebkov nadzor nad glagolskim dejanjem. Povratno osebno obliko glagola lahko razumemo kot pomožnik za tvorjenje trpnega načina, oziroma, bolje rečeno, kot trpni polpomožnik.

Ključne besede: kognitivna semantika, subjektivizacija, gramatikalizacija, glagoli zaznave, trpni način

\begin{abstract}
When (se) voir can no longer see

The article analyzes the verbs (se) voir followed by an infinitive complement as an example of subjectification according to R. W. Langacker. Subjectification refers to a semantic change involving a semantic bleaching, which can result in grammaticalization. A relatively objective construal of an entity is replaced by a more subjective one, or in other words, it designates a process in which the objectivity of the construed entity is gradually replaced by the subjectivity of the conceptualizer. The verb (se) voir reaches a relatively high degree of subjectification, in comparison with two other verbs of perception, (s') entendre, (se) sentir. (Se) voir accepts a shift in domain of perception and even a change from a domain of perception to a more abstract one. At the same time, the degree of control exercised by its subject can be to large extent attenuated. And, furthermore, its reflexive form can be regarded as a passive diathesis auxiliary, or rather as a passive diathesis semi-auxiliary.
\end{abstract}

Key words: cognitive semantics, subjectification, grammaticalization, perception verbs, diathesis 